\title{
Is HLA Typing a Diagnostic Tool in Celiac Disease?
}

\author{
Andreea L. Rachisan ${ }^{1, *}$, Alexandru Pirvan ${ }^{1}$, Nicolae Miu', Dan Gheban², Adrian Hrusca ${ }^{3}$, \\ Gabriel Samasca ${ }^{4}$, Iulia Lupan \\ ${ }^{1}$ Department of Pediatrics II, Iuliu Hatieganu University of Medicine and Pharmacy, Cluj-Napoca, Romania \\ ${ }^{2}$ Department of Pathology, Iuliu Hatieganu University of Medicine and Pharmacy, Cluj-Napoca, Romania \\ ${ }^{3}$ Department of Biophysics, Iuliu Hatieganu University of Medicine and Pharmacy, Cluj-Napoca, Romania \\ ${ }^{4}$ Department of Immunology, Iuliu Hatieganu University of Medicine and Pharmacy, Cluj-Napoca, Romania \\ ${ }^{5}$ Department of Molecular Biology, Babes-Bolyai University, Cluj-Napoca, Romania \\ *Corresponding author: andreea_rachisan@yahoo.com
}

\begin{abstract}
The "four out of five rule" has not been officially endorsed by official guidelines. However, HLAassociated genes that predispose to CD are found in as much as $30-40 \%$ of healthy people. Thus, their presence seems too indecisive for the occurrence of CD.
\end{abstract}

Keywords: celiac disease, HLA, children

Cite This Article: Andreea L. Rachisan, Alexandru Pirvan, Nicolae Miu, Dan Gheban, Adrian Hrusca, Gabriel Samasca, and Iulia Lupan, "Is HLA Typing a Diagnostic Tool in Celiac Disease?.” International Journal of Celiac Disease, vol. 4, no. 1 (2016): 9-10. doi: 10.12691/ijcd-4-1-10.

Autoimmune diseases result from an interaction between genetic predisposition and environmental triggering factors. Children suffering from an autoimmune disease such as celiac disease (CD), type 1 diabetes mellitus or Adisson disease can be at a high risk to develop another autoimmune disease [1]. This increased risk may be given by the genetic background. In this sense, there is a determinant factor for autoimmunity on the HLA region and the triggers are only responsible for which organ will be affected.

CD belongs to the group of autoimmune illnesses, with an increasing incidence especially in the pediatric population in the last years [2]. CD involves damage to the mucous membrane of the small intestine manifested by the atrophy of intestinal villi, crypt hyperplasia and intraepithelial lymphocyte infiltration caused by gluten from ingested wheat, rye and barley [3]. Genetic predisposition for CD is mainly associated with class II HLA genes of histocompatibility complex. This susceptibility is found in approximately $90-95 \%$ of patients diagnosed with the disease and are represented by HLA haplotypes Celiac $1=$ DQA1, DQB1, DR (about $40 \%$ of patients); Celiac 2 = 5q31-33; Celiac 3 = 2q33; Celiac 4 = 19p13.1 [4].

The definitive diagnosis of $\mathrm{CD}$ is made by identification of histologic changes on biopsy of the duodenum during upper gastrointestinal endoscopy while the patient maintains a gluten-containing diet [5]. An advantage in terms of HLA typing is that it is considered by the new ESPGHAN guidelines a diagnostic tool. Given the risks of pediatric intestinal biopsy under general anesthesia, it can be omitted from the CD diagnostic algorithm in patients who have high levels of TTG (10 times the normal value), EMA present in serum and at risk HLA [6]. The "four out of five rule" suggested by Catassi et al [7] proposes that the diagnosis of CD is positive in case of 4 criteria out of 5: (1) typical symptoms of CD, (2) high titer of CD antibodies, (3) positive HLA DQ2/DQ8, (4) enteropathy found on small bowel biopsy and (5) response to a glutenfree diet.

Less than $1 \%$ of patients with CD don't have the predisposing alleles. There are predisposing HLA sites in autoimmune diseases such as HLA-DR B1 03 and others that protect against autoimmune diseases (type 1 diabetes mellitus, Addison's disease) - HLA-DR B1 07; those were positive in the two presented cases. The risk HLA regarding $\mathrm{CD}$ is the HLA DQ2 (in 90\% of cases) and HLA DQ8. HLA DQ2 patients / DQ8 negative are rare, representing about $5 \%$ of those diagnosed with $\mathrm{CD}[8,9]$.

What is the role of HLA in CD? In the gut, gluten binds on HLA DQ2 and DQ8 on antigen-presenting cells leading to activation of cellular and humoral immunity. Reactive $\mathrm{T}$ lymphocytes are stimulated and they synthesize cytokines (TNF- $\alpha$ and INF- $\gamma$ ) which induce the production of metalloproteinases by fibroblasts. Metalloproteinases arrived at the brush border digest the intercellular matrix and this leads to villar atrophy, then crypt hypertrophy. On the other hand stimulated B cells produce autoantibodies that are infiltrating the mucosa [10].

Table 1. Genetic Risk from HLA-DQ2 and/or DQ8 typing [11,12]

\begin{tabular}{|l|l|}
\hline HLA DQ2/DQ8 Genotype & Risk \\
\hline DQ2+DQ8 & $1: 7(14.3 \%)$ \\
\hline DQ2+DQ2 OR DQ2 Homozygous DQB1*02 & $1: 10(10 \%)$ \\
\hline DQ8+DQ8 & $1: 12(8.4 \%)$ \\
\hline DQ8+DQB1*02 & $1: 24(4.2 \%)$ \\
\hline Homozygous DQB1*02 & $1: 26(3.8 \%)$ \\
\hline DQ2 alone & $1: 35(2.9 \%)$ \\
\hline DQ8 alone & $1: 89(1.1 \%)$ \\
\hline Population risk & $1: 100(1 \%)$ \\
\hline 1/2 DQ2: DQB1*02 & $1: 210(0.5 \%)$ \\
\hline 1/2 DQ2: DQA1 $* 05$ & $1: 1842(0.05 \%)$ \\
\hline No HLA-DQA/DQB celiac susceptibility alleles & $1: 2518(<0.04 \%)$ \\
\hline
\end{tabular}


The HLA-DQA1 and HLA-DQB1 genotypes can be determined to detect the presence or absence of the CDassociated alleles. Estimation of the degree of genetic risk for CD associated with specific HLA-DQ2/DQ8 genotypes is possible (Table 1). Absence of any CD-associated HLA alleles can exclude the diagnosis of CD [11].

There is no difference in severity of CD among patients with HLA DQ2 genotype and those with HLA DQ8 genotype. Patients with a positive DQ2 and homozygous for the HLA-DQB1*02 allele are at higher risk for CD than individuals with DQ2 and one HLA-DQB1*02 allele. Homozygosity for the HLA-DQB1*02 allele is reported to be more frequent in individuals with classic CD than in individuals with non-classic CD [14]. It is possible for individuals who have half of the DQ2 molecule (HLADQA1sequence variant [*0501 or *0505] or only the HLA-DQB1 sequence variant [*0201 or *0202]) to develop $\mathrm{CD}$, but the risk is much lower than for individuals who have the full HLA-DQ2 [15]. Other genetic influences on penetrance of CD in individuals with the HLA-DQ2 or HLA-DQ8 genotype clearly exist, as evidenced by clustering of CD in families. There several factors that can influence de genetic penetrance such as intestinal permeability [16] and innate immune response to gliadin.

In conclusion the standard of care for the diagnosis of CD includes a duodenal biopsy in all cases, with the possible exception of symptomatic children as specified by European guidelines.

\section{Abbreviations}

HLA -Human leucocyte antigen

TTG - tissue transglutaminase antibodies

EMA - antiendomisian antibodies.

\section{References}

[1] Bilbao JR1, Martín-Pagola A, Pérez De Nanclares G, Calvo B, Vitoria JC, Vázquez F, Castaño L. HLA-DRB1 and MICA in autoimmunity: common associated alleles in autoimmune disorders. Ann N Y Acad Sci. 2003; 1005: 314-8.
[2] Szalecki M. Diabetes mellitus and other autoimmunological diseases. Przegl Ped 2009; 39: 260-5.

[3] Woś H, Grzybowska-Chlebowczyk U. Coexistence of coeliac disease and diabetes type 1 in children. Ped Współcz 2009; 11: 99101.

[4] Megiorni F, Pizzuti A. HLA-DQA1 and HLA-DQB1 in Celiac disease predisposition: practical implications of the HLA molecular typing. J Biomed Sci 2012; 19:88.

[5] Lebwohl B, Rubio-Tapia A, Assiri A, Newland C, Guandalini S. Diagnosis of celiac disease. Gastrointest Endosc Clin N Am 2012; 22: 661-77.

[6] Hill ID, Horvath K. Nonbiopsy diagnosis of celiac disease: are we nearly there yet? J Pediatr Gastroenterol Nutr 2012;54: 310-1.

[7] Catassi C, Fasano A. Celiac disease diagnosis: Simpler rules are better than complicated algorithms. Am J Med 2010; 123: 691-3.

[8] Karell K, Louka AS, Moodie SJ, Ascher H, Clot F, Greco L, Ciclitira PJ, Sollid LM, Partanen J; European Genetics Cluster on Celiac Disease. HLA types in celiac disease patients not carrying the DQA1*05-DQB1*02 (DQ2) heterodimer: results from the European Genetics Cluster on Celiac Disease. Hum Immunol 2003, 64: 469-477.

[9] Samaşca G, Iancu M, Pîrvan A, Andreica M, Miu N, Constantinescu I, Cristea V, Dejica D. Histocompatibility risk antigens in celiac disease in children. Clujul Medical 2011; 84(2): 269-273.

[10] Abadie V, Sollid LM, Barreiro LB, Jabri B. Integration of genetic and immunological insights into a model of celiac disease pathogenesis. Annu Rev Immunol 2011; 29:493-525.

[11] Sollid LM, Lie BA. Celiac disease genetics: current concepts and practical applications. Clin Gastroenterol Hepatol 2005;3:843-51

[12] Megiorni F, Mora B, Bonamico M, Barbato M, Nenna R, Maiella G, Lulli P, Mazzilli MC. HLA-DQ and risk gradient for celiac disease. Hum Immunol 2009;70:55-9.

[13] Pietzak MM, Schofield TC, McGinnis FM, Nakamura RM. Stratifying risk for celiac disease in a large at-risk United States population by using HLA alleles. Clin Gastroenterol Hepatol 2009; 7: 966-71.

[14] Karinen H, Karkkalnen P, Pihlajamaki J, Janatuinen E, Heikkinen M, Julkunen R, Kosma V, Naukkarinen A, Laakso M. Gene dose effect of the DQB $1 * 0201$ allele contributes to severity of celiac disease. Scand J Gastroenterol 2006; 41: 191-9.

[15] Margaritte-Jeannin P, Babron MC, Bourgey M, Louka AS, Clot F, Percopo S, Coto I, Hugot JP, Ascher H, Sollid LM, Greco L, Clerget-Darpoux F. HLA-DQ relative risks for coeliac disease in European populations: a study of the European Genetics Cluster on Coeliac Disease. Tissue Antigens 2004; 63: 562-7.

[16] Fasano A. Zonulin and its regulation of intestinal barrier function: The biological door to inflammation, autoimmunity, and cancer. Physiol Rev 2011; 91: 151-75. 